\title{
Study on liquid sloshing characteristics of a swaying rectangular tank with a rolling baffle
}

\author{
Jing-Han Wang • Shi-Li Sun
}

Received: 14 May 2019 / Accepted: 4 September 2019 / Published online: 2 November 2019

(C) The Author(s) 2019

\begin{abstract}
This study addresses the sloshing characteristics of a liquid contained in a tank with a vertical baffle mounted at the bottom of the tank. Liquid sloshing characteristics are studied through an analytical solution procedure based on the linear velocity potential theory. The tank is forced to sway horizontally and periodically, while the baffle is fixed to the tank or rolling around a hinged point. The rectangular tank flow field is divided into a few sub-domains. The potentials are solved by a separate variable method, and the boundary conditions and matching requirements between adjacent sub-domains are used to determine the sole solution. The free surface elevations with no baffle or a low fixed baffle are compared with those in published data, and the correctness and reliability of the present method are verified. Then the baffle is forced to rotate around the bottom-mounted point. It is found that the baffle's motion, including the magnitude and the phase together, can be adjusted to suppress the free surface elevation, and even the sloshing wave can be almost eliminated.
\end{abstract}

Keywords Forced waves $\cdot$ Linear velocity potential theory $\cdot$ Rolling Baffle $\cdot$ Sloshing waves $\cdot$ Swaying tank · Wave elimination

\section{Introduction}

Sloshing problems in liquid tank have to be considered for the design of cargo ships containing fluids such as OBO, LNG, LPG and VLCC. When the tank is forced to move, the free surface of the liquid in the tank deforms, fluid moves in large amplitudes, and a resultant impact pressure is exerted on the tank walls which may damage the local structure or influence the stability of the ship. Thus, sloshing problems have attracted significant attention. Liquid sloshing leads to high local pressure and large total force, both of which are important to consider in vessel design. The two most prominent causes of severe liquid movement in a cargo tank are the resonance that happens when the sway frequency of the tank is near to or equal to the natural frequency of the liquid movement, and the movement of liquid in a tank with little or no damping. In the case of resonance, the liquid movement in the tank becomes highly magnified. With regard to damping, a rectangular tank with baffles mounted at the bottom is the common engineering remedy applied to loaded liquid containers.

J.-H. Wang · S.-L. Sun $(\varangle)$

College of Shipbuilding Engineering, Harbin Engineering University, Harbin 150001, China

e-mail: shili_sun@163.com 
Liquid sloshing can be induced by tank motion. Scholars have carried out a great deal of research on tank sloshing, and most research methods are mature. They can be divided into three approaches, namely numerical, experimental and analytical methods.

There are two main numerical schemes for addressing sloshing problems: one is to apply potential flow theory [1-3], the other is to model the viscous Navier-Stokes equations [4-6]. Both methods are extensively used to study the weakening effect of the baffles. Popov et al. [7] studied forced turning and braking of the fluid within a tank, obtained the steady-state solutions and analyzed wave height, force and overturning moment by solving continuous Navier-Stokes equations numerically. Wu et al. [8] simulated sloshing waves in a 3D liquid tank by a finite element method based on potential flow theory. The finite element method was adopted by Pal [9] to study the fluid behaviors in a flexible thin-walled cylindrical tank. On the basis of the spatially averaged Navier-Stokes equations, Liu and Lin [10] employed the large eddy simulation approach to model sloshing in a tank with bottom mounted baffles. By using the boundary element method, Wang et al. [11] studied the internal sloshing flow of a tank freely moving in an incident wave by the boundary element method. Zhao et al. [12] investigated sloshing problems in partially filled membrane tank under forced excitations, and viscous effects were considered through validation from experimental tests. Chu et al. [13] focused on mitigating effects of the number and the height of baffles mounted at tank bottom by analyzing the data from large eddy simulation and experiments.

Large displacement of the liquid in a tank produces local high stress on the walls of the vessel. Under these circumstances, the fluid motion has strong nonlinear characteristics, which presents great obstacles to theoretical analysis and numerical calculation. Some assumptions need to be introduced into the analytical or numerical procedure to render the problems solvable, but such assumptions may lead to deviation from the accurate solution. In contrast, experimental studies do not need the artificial assumptions used in numerical or analytical methods, and from this aspect, they are more reliable. Celebi and Akyildiz [14] reported experimental results to show that a shear layer and dissipated energy caused by the viscous term would form in the presence of a baffle inside the tank. They also revealed that the motion of the tank had influence on the nonlinearity of the sloshing phenomena. To analyze the pressure on tank walls and the distorted free surface, a series of experiments were undertaken by Panigrahy et al. [15]. The sway motion of the tank was controlled by a shaking table. The sway frequency of the shaking table was changed, the fill level was altered, the experimental cases with and without baffles were repeated, and the pressure and the free surface elevation were studied.

In both experimental studies and the numerical simulations, one major difficulty is tracking the deformation of the free liquid surface. From the viewpoint of an actual project, quantifying the free surface elevation in the tank is not necessary, as the most important concerns are understanding how large the natural frequencies of the tank are, and taking effective measures to avoid resonance. The resonance that occurs in a liquid cargo tank and effectiveness of mechanisms for suppressing liquid sloshing can be well characterized by analytical methods. Compared with the experimental method and the numerical method, the analytical method can easily give the relationship between physical quantities, and has the advantages of fast calculation speed and small error. Abramson [16] applied linear potential flow theories to analyze the liquid motion in cylindrical and spherical tanks. Evans and Mciver [17] studied the influence of a vertical baffle on the resonant frequency of the fluid in a rectangular container by linear wave theory. The accuracy of the simple approximation is evaluated by comparing it with the exact solution based on eigenfunction expansions. It was found that a surface-piercing baffle can significantly change the resonant frequencies, while the effect of a bottom-mounted baffle is usually negligible. Wu [18] employed velocity potential theory to study the second-order resonance of sloshing. Zhou [19] deduced the analytical solution of the wave radiated by a bottomopened rectangular body floating in water of finite depth. Zhang et al. [20] found that floating foams can weaken both the sloshing amplitude and the hydrodynamic pressure through observations from experiments and an analytical potential-flow solution.

Recent focus has shifted from studying hydrodynamic behaviors of liquid in a tank to seeking methods of weakening the sloshing phenomena, for which baffles are the most frequently used remedy [21-25]. Although the present topic has been widely researched, most of the previous work has considered fixed baffles, and sloshing was mitigated by changing the baffles' height, number or altering the baffles' shape. In the present paper, the baffle is rotating around a bottom-mounted point while the tank is swaying horizontally. The baffle's motion, including the 


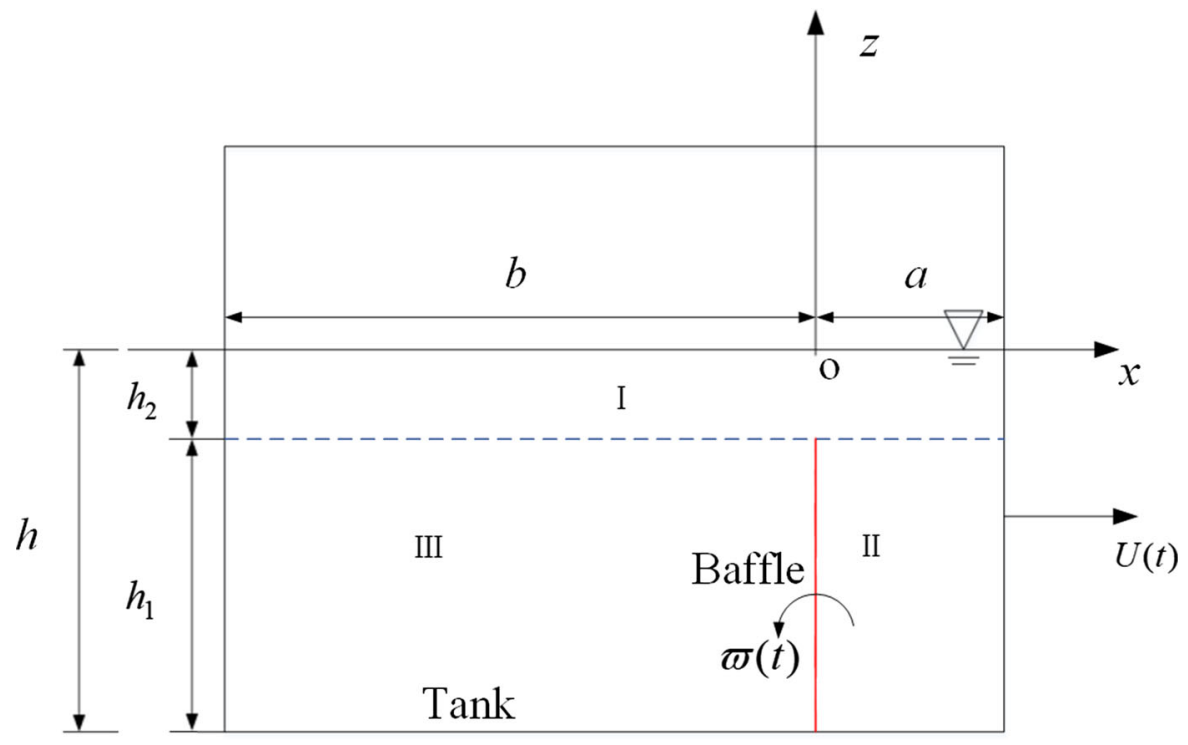

Fig. 1 Sketch map

amplitude and the phase, are adjusted to weaken the sloshing. The mitigating effect is remarkable, and the wave can even be almost eliminated. In this paper, separation of variables is utilized to solve the governing equation, together with all the boundary conditions, to obtain analytical results. The natural frequencies of the tank with the baffle are also computed by fast fourier transform (FFT). Liquid sloshing characteristics in a tank with and without baffles are compared. The effects of the baffle parameters, such as height, on sloshing are examined.

\section{Governing equation and boundary conditions}

A two-dimensional rectangular tank model is shown in Fig. 1. The tank is undergoing horizontal oscillation with displacement $X(\mathrm{t})$, the total length of the rectangular tank is $2 l$, the height from the free liquid surface to the bottom of the tank is taken as $h$, and a rigid baffle with a height of $h_{1}$ is fixed to the bottom of the tank. The distance from the rigid baffle to the right wall is $a$, and to the left is $b$. In the coordinate system, the $z$-axis is vertical and the $x$-axis is horizontal and is coincident with the baffle line.

Viscosity and compressibility of the fluid are neglected, rotational behavior of the flow is overlooked, the motion of the tank and the baffle is assumed to be small, and the fluid field satisfies the Laplace equation [17,21,23].

$\nabla^{2} \phi(x, z, t)=0$.

To seek a unique solution, the boundary conditions and initial conditions in the fluid domain are needed. The impermeable bottom condition conforms with

$\left.\frac{\partial \phi}{\partial z}\right|_{z=-h}=0$.

On the side wall of the tank, we have

$\left.\frac{\partial \phi}{\partial n}\right|_{x=-b, x=a}=\boldsymbol{U} \cdot \boldsymbol{n}$.

On the baffle,

$\left.\frac{\partial \phi}{\partial n}\right|_{x=0,-h<z<-h_{2}}=(\boldsymbol{U}+\Lambda \times \boldsymbol{r}) \cdot \boldsymbol{n}$, 
where $\boldsymbol{U}$ denotes the translational velocity of the tank, $\boldsymbol{\Lambda}$ represents the rotational speed, and $\mathbf{r}$ is the position vector to the hinged point of the baffle. According to the Bernoulli equation, the dynamic free surface condition can be expressed as $[18,26]$

$\eta=-\frac{1}{g}\left(\frac{\partial \phi}{\partial t}+\frac{1}{2} \nabla \phi \cdot \nabla \phi\right)$

where $g$ is the gravity acceleration. Equation (6) is transformed by Taylor expansion, ignoring the higher order term, and the linearized free surface condition is obtained

$\eta=-\frac{1}{g} \frac{\partial \phi}{\partial t}, \quad z=0$.

The kinematic condition of the free surface is written as

$\frac{\partial \eta}{\partial t}=\frac{\partial \phi}{\partial z}, \quad z=0$.

Substitute Eq. (6) into Eq. (7), and we have

$\frac{\partial^{2} \phi}{\partial t^{2}}+g \frac{\partial \phi}{\partial z}=0, \quad z=0$

Initially the free surface is assumed to be flat

$\eta(x, t)=0, \quad z=0, \quad t=0$,

and the potential and its time derivative are zero

$\phi(x, z, t)=0 \quad$ and $\quad \frac{\partial \phi}{\partial t}(x, z, t)=0, \quad z=0, \quad t=0$.

\section{Sloshing of a swaying liquid tank with a fixed baffle}

\subsection{Expressions for potential in the fixed baffle case}

We first consider an example of a liquid tank with swaying motion $U(t)=U_{0} \sin \Omega_{0} t$, where the baffle is fixed to the tank. To give the analytical solution, the fluid in the rectangular tank is divided into three sub-domains as shown in Fig. 1, which are $\mathrm{I}\left(-b \leq x \leq a,-h_{2} \leq z \leq 0\right)$, II $\left(0 \leq x \leq a,-h \leq z \leq-h_{2}\right)$ and III $\left(-b \leq x \leq 0,-h \leq z \leq-h_{2}\right)$ respectively. Similar work can be seen in Choun and Yun [27] and Zhou, Wu and Zhang [19], who also divided the fluid domain into a few sub-regions, but they focused on the frequency-domain solution. The potentials in each sub-domain are represented by $\phi_{1}, \phi_{2}$ and $\phi_{3}$. These potentials satisfy

I $\left\{\begin{array}{l}\nabla^{2} \phi_{1}=0, \\ \left.\frac{\partial \phi_{1}}{\partial x}\right|_{x=a}=U(t), \quad \text { II } \\ \left.\frac{\partial \phi_{1}}{\partial x}\right|_{x=-b}=U(t),\end{array} \quad\left\{\begin{array}{l}\nabla^{2} \phi_{2}=0, \\ \left.\frac{\partial \phi_{2}}{\partial x}\right|_{x=a}=U(t), \\ \left.\frac{\partial \phi_{2}}{\partial x}\right|_{x=0}=U(t), \\ \left.\frac{\partial \phi_{2}}{\partial z}\right|_{z=-h}=0,\end{array} \quad\right.\right.$ III $\left\{\begin{array}{l}\nabla^{2} \phi_{3}=0, \\ \left.\frac{\partial \phi_{3}}{\partial x}\right|_{x=-b}=U(t), \\ \left.\frac{\partial \phi_{3}}{\partial x}\right|_{x=0}=U(t), \\ \left.\frac{\partial \phi_{3}}{\partial z}\right|_{z=-h}=0 .\end{array}\right.$

By separating variables, the velocity potential can be written as infinite series [19,27]

$$
\begin{aligned}
& \phi_{1}=U(t) x+\sum_{n=1}^{\infty}\left[A_{n}(t) \cosh k_{n}\left(z+h_{2}\right)+B_{n}(t) \sinh k_{n}\left(z+h_{2}\right)\right] \cos k_{n}(x+b), \\
& \phi_{2}=U(t) x+\sum_{n=1}^{\infty} C_{n}(t) \cos \lambda_{n}(x-a) \cosh \lambda_{n}(z+h), \\
& \phi_{3}=U(t) x+\sum_{n=1}^{\infty} D_{n}(t) \cos \gamma_{n}(x+b) \cosh \gamma_{n}(z+h),
\end{aligned}
$$


where

$k_{n}=\frac{n \pi}{a+b}, \quad \lambda_{n}=\frac{n \pi}{a} \quad \gamma_{n}=\frac{n \pi}{b}, \quad n=1,2,3 \ldots$

The unique and unknown coefficients in Eqs. (12)-(14) are to be fixed by continuous conditions between different regions as well as the free surface condition.

\subsection{Solution for the unknown coefficients in the fixed baffle case}

Along the interfaces between adjacent fluid domains, matching conditions are applied to ensure velocity and pressure continuity $[19,28,29]$. Thus at each interface $z=-h_{2}$ and on the free surface $z=0$, we have

$$
\begin{aligned}
& \phi_{1}=\phi_{2}\left(0 \leq x \leq a, z=-h_{2}\right), \\
& \phi_{1}=\phi_{3}\left(-b \leq x \leq 0, z=-h_{2}\right), \\
& \frac{\partial \phi_{1}}{\partial z}=\left\{\begin{array}{l}
\frac{\partial \phi_{2}}{\partial z}\left(0 \leq x \leq a, z=-h_{2}\right), \\
\frac{\partial \phi_{3}}{\partial z}\left(-b \leq x \leq 0, z=-h_{2}\right),
\end{array}\right. \\
& \frac{\partial^{2} \phi_{1}}{\partial t^{2}}+g \frac{\partial \phi_{1}}{\partial z}=0(z=0) .
\end{aligned}
$$

At the initial moment, we have

$$
\begin{aligned}
& \phi_{1}=\phi_{2}\left(0 \leq x \leq a, z=-h_{2}, t=0\right), \quad \frac{\partial \phi_{1}}{\partial t}=\frac{\partial \phi_{2}}{\partial t}\left(0 \leq x \leq a, z=-h_{2}, t=0\right), \\
& \phi_{1}=\phi_{3}\left(-b \leq x \leq 0, z=-h_{2}, t=0\right), \quad \frac{\partial \phi_{1}}{\partial t}=\frac{\partial \phi_{3}}{\partial t}\left(-b \leq x \leq 0, z=-h_{2}, t=0\right) \text {, } \\
& \begin{array}{l}
\frac{\partial \phi_{1}}{\partial z}=\left\{\begin{array}{l}
\frac{\partial \phi_{2}}{\partial z}\left(0 \leq x \leq a, z=-h_{2}, t=0\right), \\
\frac{\partial \phi_{3}}{\partial z}\left(-b \leq x \leq 0, z=-h_{2}, t=0\right),
\end{array}\right. \\
\phi_{1}(x, 0,0)=0,
\end{array} \quad \frac{\partial^{2} \phi_{1}}{\partial z \partial t}=\left\{\begin{array}{l}
\frac{\partial^{2} \phi_{2}}{\partial z \partial t}\left(0 \leq x \leq a, z=-h_{2}, t=0\right), \\
\frac{\partial^{2} \phi_{3}}{\partial z \partial t}\left(-b \leq x \leq 0, z=-h_{2}, t=0\right),
\end{array}\right. \\
& \frac{\partial \phi_{1}}{\partial t}(x, 0,0)=0 .
\end{aligned}
$$

Equations (12)-(14) are substituted into Eq. (15), and the orthogonality of the trigonometric function in the eigenfunction method is used $[18,20]$; we then have the relationship between the unknown coefficient expressions

$$
\begin{aligned}
& \sum_{j=1}^{\infty} A_{j}(t) a_{j i}=\frac{a}{2} C_{i}(t) \cosh \lambda_{i} h_{1}, \\
& \sum_{j=1}^{\infty} A_{j}(t) b_{j i}=\frac{b}{2} D_{i}(t) \cosh \gamma_{i} h_{1}, \\
& \frac{a+b}{2} k_{i} B_{i}(t)=\sum_{j=1}^{\infty} \lambda_{j} C_{j}(t) a_{i j} \sinh \lambda_{j} h_{1}+\sum_{j=1}^{\infty} \gamma_{j} D_{j}(t) b_{i j} \sinh \gamma_{j} h_{1}, \\
& U^{\prime \prime}(t) c_{i}+\frac{a+b}{2}\left[A_{i}^{\prime \prime}(t) \cosh k_{i} h_{2}+B_{i}^{\prime \prime}(t) \sinh k_{i} h_{2}\right]+\frac{a+b}{2} g k_{i}\left[A_{i}(t) \sinh k_{i} h_{2}+B_{i}(t) \cosh k_{i} h_{2}\right]=0,
\end{aligned}
$$

where

$$
\begin{aligned}
& a_{j i}=\int_{0}^{a} \cos k_{j}(x+b) \cdot \cos \lambda_{i}(x-a) \mathrm{d} x, \\
& b_{j i}=\int_{-b}^{0} \cos k_{j}(x+b) \cdot \cos \gamma_{i}(x+b) \mathrm{d} x, \quad i=1,2,3 \ldots ; \quad j=1,2,3 \ldots \\
& c_{i}=\int_{-b}^{a} x \cos k_{i}(x+b) \mathrm{d} x .
\end{aligned}
$$

Gaussian elimination method is used. The Laplace transform is performed on Eq. (20) [30], and then we have

$$
B_{i}(t)=\frac{2}{(a+b) k_{i}} \sum_{m=1}^{\infty} A_{m}(t) \cdot P_{i m},
$$


$A_{i}(t)=Q_{i}(t)+\left(\omega_{i} \tanh k_{i} h_{2}-\frac{g k_{i}}{\omega_{i}}\right) \int_{0}^{t} B_{i}(\tau) \sin \omega_{i}(t-\tau) \mathrm{d} \tau-B_{i}(t) \tanh k_{i} h_{2}$,

where

$$
\begin{aligned}
P_{i m}= & \sum_{j=1}^{\infty}\left(\frac{2 \lambda_{j} a_{m j} a_{i j} \tanh \left(\lambda_{j} h_{1}\right)}{a}+\frac{2 \gamma_{j} b_{m j} b_{i j} \tanh \left(\gamma_{j} h_{1}\right)}{b}\right), \\
\omega_{i}= & \sqrt{g k_{i} \tanh k_{i} h_{2}}, \\
Q_{i}(t)= & \frac{-2 c_{i}}{(a+b) \omega_{i} \cosh k_{i} h_{2}} \int_{0}^{t} U^{\prime \prime}(\tau) \sin \omega_{i}(t-\tau) \mathrm{d} \tau+\frac{\sin \omega_{i} t}{\omega_{i}} A_{i}^{\prime}(0)+\frac{\tanh k_{i} h_{2}}{\omega_{i}} B_{i}^{\prime}(0) \sin \omega_{i} t \\
& +A_{i}(0) \cos \omega_{m} t+B_{i}(0) \cos \left(\omega_{i} t\right) \tanh \left(k_{i} h_{2}\right) .
\end{aligned}
$$

Substitute Eqs. (22) into (21), and finally the expression of the unknown coefficient $B_{i}(t)$ is obtained

$$
\begin{aligned}
\left(\frac{a+b}{2} k_{i}+P_{i i} \tanh k_{i} h_{2}\right) B_{i}(t)= & \sum_{m=1}^{\infty}\left[Q_{m}(t)+\left(\omega_{m} \tanh k_{m} h_{2}-\frac{g k_{m}}{\omega_{m}}\right) \int_{0}^{t} B_{m}(\tau) \sin \omega_{m}(t-\tau) \mathrm{d} \tau\right] \cdot P_{i m} \\
& -\sum_{m=1, m \neq i}^{\infty} B_{m}(t) \tanh \left(k_{m} h_{2}\right) \cdot P_{i m}
\end{aligned}
$$

Equation (26), which is to be solved by the iteration method, involves some infinite series, and thus for practical computation, it must be truncated. After $B_{i}(t)$ is solved, $A_{i}(t), C_{i}(t)$ and $D_{i}(t)$ can be obtained in turn. Equations (12)-(14) are substituted into Eq. (16), $A_{i}(0), A_{i}^{\prime}(0), B_{i}(0), B_{i}^{\prime}(0), C_{i}(0), C_{i}^{\prime}(0), D_{i}(0), D_{i}^{\prime}(0)$ can be also obtained by the above steps.

\section{Sloshing of a swaying liquid tank with a rolling baffle}

4.1 Expression for the potential in the rolling baffle case

Consider the case in which the tank is swaying with $U(t)=U_{0} \sin \Omega_{0} t$, the baffle at the bottom of the tank rotates around the point $(0,-\mathrm{h})$ under the external excitation of frequency $\Omega_{\mathrm{p}}$, and does a micro-amplitude roll motion with angular velocity $\varpi(t)=\varpi_{0} \sin \left(\Omega_{\mathrm{p}} t+\varphi\right)$. Then the control equations and boundary conditions satisfied in each sub-domain can be listed as [27]

$$
\text { I }\left\{\begin{array} { l } 
{ \nabla ^ { 2 } \phi _ { 1 } = 0 , } \\
{ \frac { \partial \phi _ { 1 } } { \partial x } | _ { x = a } = U ( t ) , } \\
{ \frac { \partial \phi _ { 1 } } { \partial x } | _ { x = - b } = U ( t ) , }
\end{array} \quad \text { II } \left\{\begin{array} { l } 
{ \nabla ^ { 2 } \phi _ { 2 } = 0 , } \\
{ \frac { \partial \phi _ { 2 } } { \partial x } | _ { x = a } = U ( t ) , } \\
{ \frac { \partial \phi _ { 2 } } { \partial x } | _ { x = 0 } = U ( t ) + \varpi ( t ) \cdot ( z + h ) , } \\
{ \frac { \partial \phi _ { 2 } } { \partial z } | _ { z = - h } = 0 , }
\end{array} \quad \text { III } \left\{\begin{array}{l}
\nabla^{2} \phi_{3}=0, \\
\left.\frac{\partial \phi_{3}}{\partial x}\right|_{x=-b}=U(t), \\
\left.\frac{\partial \phi_{3}}{\partial x}\right|_{x=0}=U(t)+\varpi(t) \cdot(z+h), \\
\left.\frac{\partial \phi_{3}}{\partial z}\right|_{z=-h}=0 .
\end{array}\right.\right.\right.
$$

Based on a similar procedure in Sect. 3, the potential in each domain is expressed as [19,27]

$$
\begin{aligned}
\phi_{1}= & U(t) x+\sum_{n=1}^{\infty}\left[A_{n}(t) \cosh k_{n}\left(z+h_{2}\right)+B_{n}(t) \sinh k_{n}\left(z+h_{2}\right)\right] \cos k_{n}(x+b), \\
\phi_{2}= & U(t) x-\frac{\varpi(t)}{2 a}\left[(z+h)(x-a)^{2}-\frac{(z+h)^{3}}{3}\right] \\
& +\sum_{n=1}^{\infty}\left[C_{n}(t) \cosh \lambda_{n}(z+h)+D_{n}(t) \sinh \lambda_{n}(z+h)\right] \cos \lambda_{n}(x-a), \\
\phi_{3}= & U(t) x+\frac{\varpi(t)}{2 b}\left[(z+h)(x+b)^{2}-\frac{(z+h)^{3}}{3}\right]
\end{aligned}
$$




$$
+\sum_{n=1}^{\infty}\left[E_{n}(t) \cosh \gamma_{n}(z+h)+F_{n}(t) \sinh \gamma_{n}(z+h)\right] \cos \gamma_{n}(x+b)
$$

where

$k_{n}=\frac{n \pi}{a+b}, \quad \lambda_{n}=\frac{n \pi}{a}, \quad \gamma_{n}=\frac{n \pi}{b}$.

4.2 Solution for the unknown coefficients in the rolling baffle case

Using the continuous condition at $z=-h_{2}[19,28]$, the free surface condition $z=0$ and the bottom boundary conditions $z=-h$, we have

$$
\begin{aligned}
& \phi_{1}=\phi_{2}\left(0 \leq x \leq a, z=-h_{2}\right), \\
& \phi_{1}=\phi_{3}\left(-b \leq x \leq 0, z=-h_{2}\right), \\
& \frac{\partial \phi_{1}}{\partial z}=\left\{\begin{array}{l}
\frac{\partial \phi_{2}}{\partial z}\left(0 \leq x \leq a, z=-h_{2}\right), \\
\frac{\partial \phi_{3}}{\partial z}\left(-b \leq x \leq 0, z=-h_{2}\right),
\end{array}\right. \\
& \frac{\partial^{2} \phi_{1}}{\partial t^{2}}+g \frac{\partial \phi_{1}}{\partial z}=0(z=0), \\
& \frac{\partial \phi_{2}}{\partial z}=0(0 \leq x \leq a, z=-h), \\
& \frac{\partial \phi_{3}}{\partial z}=0(-b \leq x \leq 0, z=-h) .
\end{aligned}
$$

Substituting Eqs. (28-30) into Eq. (31) and then using the orthogonality of the trigonometric functions [18,20], we have

$$
\begin{aligned}
& \sum_{j=1}^{\infty} A_{j}(t) a_{j i}=-\frac{\varpi(t)}{2 a} d_{i}+\frac{a}{2}\left[C_{i}(t) \cosh \lambda_{i} h_{1}+D_{i}(t) \sinh \lambda_{i} h_{1}\right], \\
& \sum_{j=1}^{\infty} A_{j}(t) b_{j i}=\frac{\varpi(t)}{2 b} e_{i}+\frac{b}{2}\left[E_{i}(t) \cosh \gamma_{i} h_{1}+F_{i}(t) \sinh \gamma_{i} h_{1}\right], \\
& \frac{(a+b) B_{i}(t) k_{i}}{2}=-\frac{\varpi(t)}{2 a} f_{i}+\sum_{j=1}^{\infty}\left[C_{j}(t) \lambda_{j} \sinh \lambda_{j} h_{1}+D_{j}(t) \lambda_{j} \cosh \lambda_{j} h_{1}\right] a_{i j} \\
& \quad+\frac{\varpi(t)}{2 b} g_{i i}+\sum_{j=1}^{\infty}\left[E_{j}(t) \gamma_{j} \sinh \gamma_{j} h_{1}+F_{j}(t) \gamma_{j} \cosh \gamma_{j} h_{1}\right] b_{i j}, \\
& \quad+\frac{a+b}{2} g k_{i}\left[A_{i}(t) \sinh k_{i} h_{2}+B_{i}(t) \cosh k_{i} h_{2}\right]=0, \\
& -\frac{\varpi(t)}{2 a} r_{i}+\frac{a D_{i}(t) \lambda_{i}}{2}=0, \\
& \frac{\varpi(t)}{2 b} s_{i}+\frac{b F_{i}(t) \gamma_{i}}{2}=0,
\end{aligned}
$$


where

$$
\begin{aligned}
a_{j i} & =\int_{0}^{a} \cos k_{j}(x+b) \cdot \cos \lambda_{i}(x-a) \mathrm{d} x, \\
b_{j i} & =\int_{-b}^{0} \cos k_{j}(x+b) \cdot \cos \gamma_{i}(x+b) \mathrm{d} x, \\
c_{i} & =\int_{-b}^{a} x \cos k_{i}(x+b) \mathrm{d} x, \\
d_{i} & =\int_{0}^{a}\left[h_{1}(x-a)^{2}-\frac{h_{1}^{3}}{3}\right] \cos \lambda_{i}(x-a) \mathrm{d} x, \\
e_{i} & =\int_{-b}^{0}\left[h_{1}(x+b)^{2}-\frac{h_{1}^{3}}{3}\right] \cos \gamma_{i}(x+b) \mathrm{d} x, \\
f_{i} & =\int_{0}^{a}\left[(x-a)^{2}-h_{1}^{2}\right] \cos k_{i}(x+b) \mathrm{d} x, \\
g_{i} & =\int_{-b}^{0}\left[(x+b)^{2}-h_{1}^{2}\right] \cos k_{i}(x+b) \mathrm{d} x, \\
r_{i} & =\int_{0}^{a}(x-a)^{2} \cos \lambda_{i}(x-a) \mathrm{d} x, \\
s_{i} & =\int_{-b}^{0}(x+b)^{2} \cos \gamma_{i}(x+b) \mathrm{d} x .
\end{aligned}
$$

Equation (32) is solved by Gaussian elimination, and we have

$$
\begin{aligned}
P_{i} B_{i}(t)= & -\frac{\varpi(t)}{2 a} f_{i}+\frac{\varpi(t)}{2 b} g_{i} \\
& +\sum_{j=1}^{\infty} \frac{2 \lambda_{j} a_{i j} \tanh \lambda_{j} h_{1}}{a} D_{j}(t) a_{i j} \lambda_{j} \cosh \lambda_{j} h_{1}+\sum_{j=1}^{\infty} \frac{2 \gamma_{j} b_{i j} \tanh \gamma_{j} h_{1}}{b} F_{j}(t) b_{i j} \gamma_{j} \cosh \gamma_{j} h_{1} \\
& +\sum_{j=1}^{\infty} \frac{2 \lambda_{j} a_{i j} \tanh \lambda_{j} h_{1}}{a}\left[\sum_{m=1}^{\infty}\left(Q_{m}(t)+\left(\omega_{m} \tanh k_{m} h_{2}-\frac{g k_{m}}{\omega_{m}}\right) \int_{0}^{t} B_{m}(\tau) \sin \omega_{m}(t-\tau) \mathrm{d} \tau\right) a_{m j}\right] \\
& -\sum_{j=1}^{\infty} \frac{2 \lambda_{j} a_{i j} \tanh \lambda_{j} h_{1}}{a}\left[\sum_{m=1, m \neq i}^{\infty} B_{m}(t) a_{m j} \tanh k_{m} h_{2}+\frac{\varpi(t)}{2 a} d_{j j}-\frac{a}{2} D_{j}(t) \sinh \lambda_{j} h_{1}\right] \\
& +\sum_{j=1}^{\infty} \frac{2 \gamma_{j} b_{i j} \tanh \gamma_{j} h_{1}}{b}\left[\sum_{m=1}^{\infty}\left(Q_{m}(t)+\left(\omega_{m} \tanh k_{m} h_{2}-\frac{g k_{m}}{\omega_{m}}\right) \int_{0}^{t} B_{m}(\tau) \sin \omega_{m}(t-\tau) \mathrm{d} \tau\right) b_{m j}\right] \\
& -\sum_{j=1}^{\infty} \frac{2 \lambda_{j} a_{i j} \tanh \lambda_{j} h_{1}}{a}\left[\sum_{m=1, m \neq i}^{\infty} B_{m}(t) b_{m j} \tanh k_{m} h_{2}-\frac{\varpi(t)}{2 b} e_{j j}-\frac{b}{2} F_{j}(t) \sinh \gamma_{j} h_{1}\right],
\end{aligned}
$$

where

$$
\begin{aligned}
P_{i}= & \frac{(a+b) k_{i}}{2}+\tanh \left(k_{i} h_{2}\right) \sum_{j=1}^{\infty}\left(\frac{2 \lambda_{j} \tanh \lambda_{j} h_{1}}{a} a_{i j}^{2}+\frac{2 \gamma_{j} \tanh \gamma_{j} h_{1}}{b} b_{i j}^{2}\right) \\
Q_{m}(t)= & \frac{-2 c_{m m}}{(a+b) \omega_{m} \cosh k_{m} h_{2}} \int_{0}^{t} U^{\prime \prime}(\tau) \sin \omega_{m}(t-\tau) \mathrm{d} \tau+\frac{\sin \omega_{m} t}{\omega_{m}} A_{m}^{\prime}(0)+\frac{\tanh k_{m} h_{2}}{\omega_{m}} B_{m}^{\prime}(0) \sin \omega_{m} t \\
& +A_{m}(0) \cos \omega_{m} t+B_{m}(0) \cos \left(\omega_{m} t\right) \tanh \left(k_{m} h_{2}\right) .
\end{aligned}
$$


When the Laplace transform is performed on Eq. (32) [30], we have a relational expression as follows:

$A_{i}(t)=Q_{i}(t)+\left(\omega_{i} \tanh k_{i} h_{2}-\frac{g k_{i}}{\omega_{i}}\right) \int_{0}^{t} B_{i}(\tau) \sin \omega_{i}(t-\tau) \mathrm{d} \tau-B_{i}(t) \tanh k_{i} h_{2}$,

where $\omega_{i}=\sqrt{g k_{i} \tanh k_{i} h_{2}}$.

$$
\begin{aligned}
Q_{i}(t)= & \frac{-2 c_{i}}{(a+b) \omega_{i} \cosh k_{i} h_{2}} \int_{0}^{t} U^{\prime \prime}(\tau) \sin \omega_{i}(t-\tau) \mathrm{d} \tau+\frac{\sin \omega_{i} t}{\omega_{i}} A_{i}^{\prime}(0)+\frac{\tanh k_{i} h_{2}}{\omega_{i}} B_{i}^{\prime}(0) \sin \omega_{i} t \\
& +A_{i}(0) \cos \omega_{m} t+B_{i}(0) \cos \left(\omega_{i} t\right) \tanh \left(k_{i} h_{2}\right) .
\end{aligned}
$$

We can get the approximate solution of $B_{i}(t)$ by the iteration algorithm. Then we can get $A_{i}(t)$ via the relationship of coefficients. The expressions of $C_{i}(t), D_{i}(t), E_{i}(t), F_{i}(t)$ can be obtained by solving Eq. (32).

$$
\begin{aligned}
C_{i}(t) & =\frac{2}{a \cosh \lambda_{i} h_{1}}\left(\sum_{j=1}^{\infty} A_{j}(t) a_{j i}+\frac{\varpi(t)}{2 a} d_{i}-\frac{a}{2} D_{i}(t) \sinh \lambda_{i} h_{1}\right), \\
E_{i}(t) & =\frac{2}{b \cosh \gamma_{i} h_{1}}\left(\sum_{j=1}^{\infty} A_{j}(t) b_{j i}-\frac{\varpi(t)}{2 b} e_{i}-\frac{b}{2} F_{i}(t) \sinh \gamma_{i} h_{1}\right), \\
D_{i}(t) & =\frac{\varpi(t) r_{i}}{a^{2} \lambda_{i}} \\
F_{i}(t) & =-\frac{\varpi(t) s_{i}}{b^{2} \gamma_{i}} .
\end{aligned}
$$

The free surface is undisturbed initially, or $z=\eta(x, 0)=0$. Thus, we can get the initial value of coefficients by solving $\phi_{1}(x, 0,0)=0$ and $\partial \phi_{1}(x, 0,0) / \partial t=0$.

\section{Analytical results}

\subsection{Convergence study with the number of the series}

The potential expressions are represented by infinite series, and they must be truncated. Convergence analysis with respect to series number $N$ is performed here. The size and parameters of the tank are $a=b=l=h=0.7 \mathrm{~m}$. The motion can be expressed as $X=-X_{0} \cos \Omega_{0} t$ and the external excitation frequency is $\Omega_{0}=1 \mathrm{rad} / \mathrm{s}$. The baffle's height $h_{1} / h$ is taken as $0.3,0.5,0.7,0.9$, respectively. The results with $N=15$ and $N=50$ are presented in Fig. 2, which shows that they are in very good agreement. This means $N=15$ can provide sufficiently accurate results; therefore, in the following discussion, the potential expressions are truncated with 15 series, unless otherwise stated. When $h_{1} / h$ increases from 0.3 to 0.9 , the characteristic frequency $\omega_{1}$ in Eq. (24) decreases from 4.2 to 1.85 , and they all are much larger than the excitation frequency $\Omega_{0}=1 \mathrm{rad} / \mathrm{s}$ and thus no resonance happens. In range of $\omega_{1} / \Omega_{0}$ changing from 4.2 to 1.85 , the influence of the characteristic frequency $\omega_{1}$ on the value of free surface elevation is relatively smaller.

\subsection{Verification of the results}

The accuracy of the above process in solving the velocity potential coefficients is verified via the identity relationship of equations. Generally, the natural frequency of the sloshing fluid in a rectangular tank without a baffle can be estimated according to the linear theoretical formula derived by $\mathrm{Wu}[18]$ :

$\Omega_{n}=\sqrt{\frac{n g \pi}{a+b} \tanh \left(\frac{n \pi h}{a+b}\right)}$. 


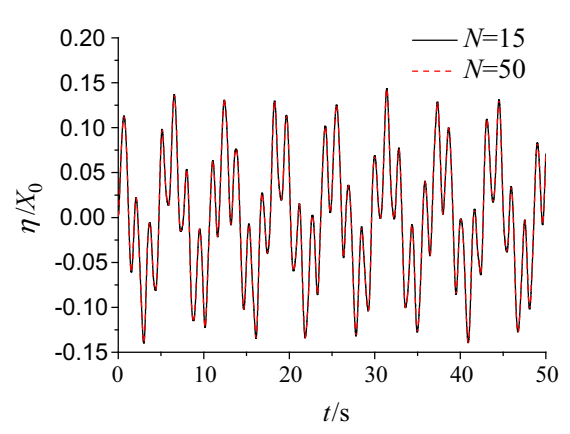

(a) $h_{1} / h=0.3, \omega_{1}=4.20$

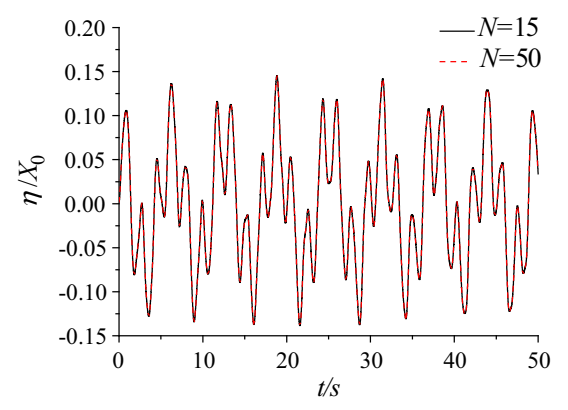

(c) $h_{1} / h=0.7, \omega_{1}=3.11$

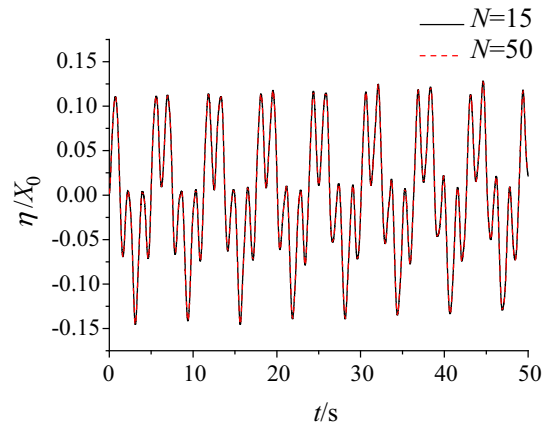

(b) $h_{1} / h=0.5, \omega_{1}=3.80$

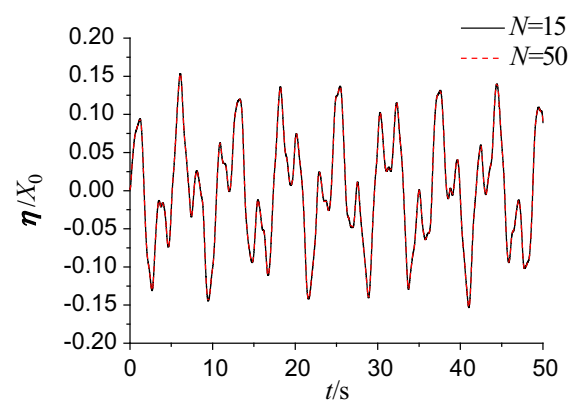

(d) $h_{1} / h=0.9, \omega_{1}=1.85$

Fig. 2 Free surface elevation $\eta$ with different baffle's height, the sway frequency of the tank $\Omega_{0}=1 \mathrm{rad} / \mathrm{s}$

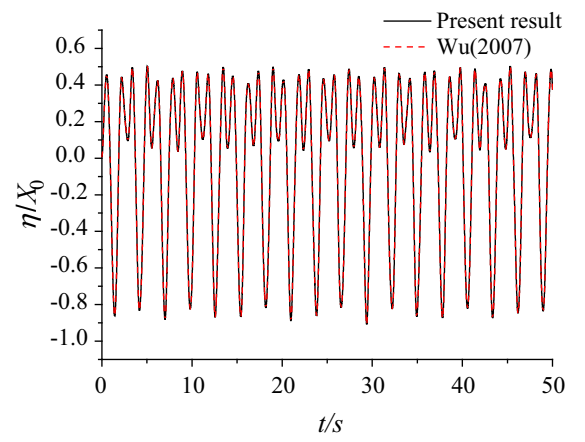

(a) $\Omega_{0}=0.5 \Omega_{1}$

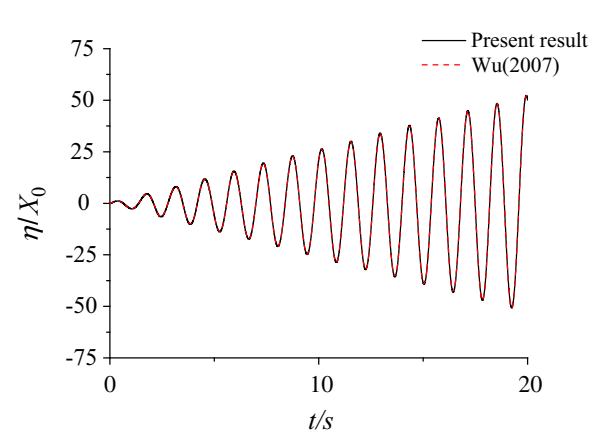

(b) $\Omega_{0}=\Omega_{1}$

Fig. 3 Comparison of free surface elevation $\eta$ between the present result and that by $\mathrm{Wu}[18]$ with no baffle

The calculation size and parameters of the tank are $a=b=l=h=0.7 \mathrm{~m}$ with no baffle $h_{1}=0$. According to Eq. (37), it can be known that the first natural frequency or the fundamental frequency $\Omega_{1}=4.4910 \mathrm{rad} / \mathrm{s}$ under this condition. The external excitation frequency is chosen to be equal to and far from the natural frequency for analysis, or $\Omega_{0}=\Omega_{1}, \Omega_{0}=0.5 \Omega_{1}$. Figure 3 shows the free surface elevation on the left wall of the tank $x=-b$ with excitation frequency $\Omega_{0}=0.5 \Omega_{1}$ and $\Omega_{1}$. In the absence of a baffle, the wave height obtained by the present method completely coincides with that by $\mathrm{Wu}[18]$.

Then the case of $h_{1} / h=0.1$ is considered, in which the baffle's height is very small, and other geometric parameters of the tank are as the above. Theoretically, if the baffle's height changes a little, the influence of the baffle's rise on the wave is also small. Here, $\Omega_{0}=0.5 \Omega_{1}$ is used. Figure 4 shows the corresponding free surface 
Fig. 4 Comparison of free surface elevation $\eta$ between the present result with a low baffle and that by $\mathrm{Wu}[18]$ with no baffle, $\Omega_{0}=0.5 \Omega_{1}$
Fig. 5 Change of free surface elevation $\eta$ with time $t$ at $x=-b$, the baffle height changes from 0.2 to $0.9 \mathrm{~h}$, the sway frequency of the tank $\Omega_{0}=1.002 \Omega_{1}$
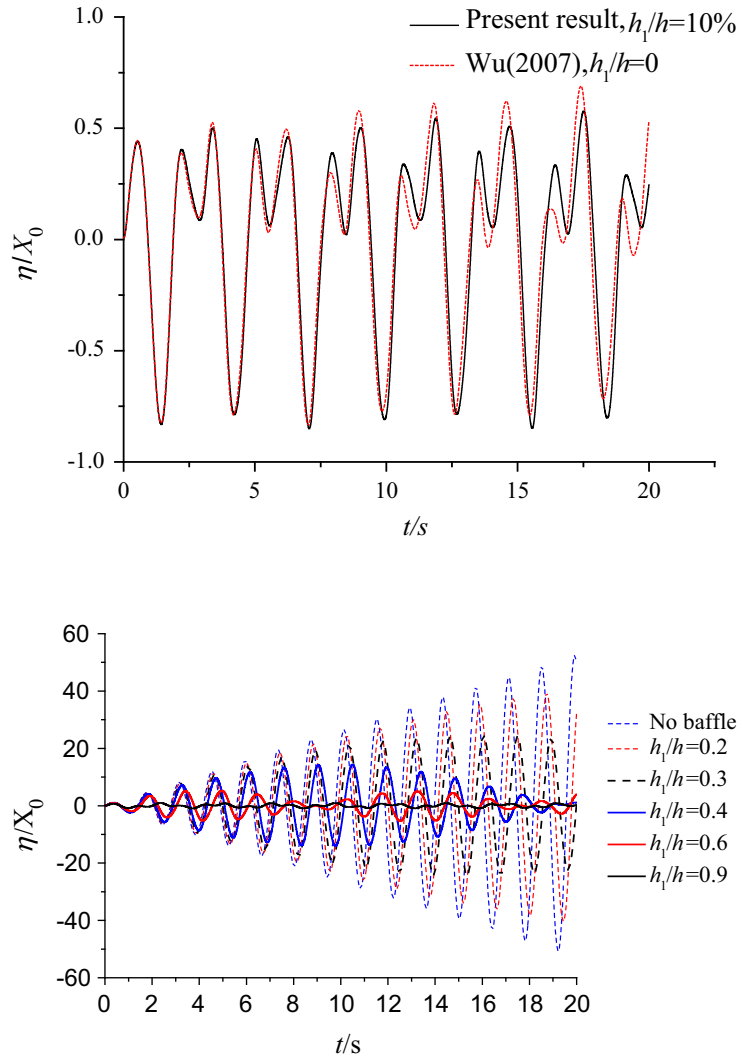

elevation. Comparing the present result with a very short baffle and that by $\mathrm{Wu}$ [18] with no baffle, they are very close to each other, which conforms with the expectation.

\subsection{Influence of baffle height on wave elevation}

When excitation frequency $\Omega_{0}=1.002 \Omega_{1}$, then large amplitude sloshing will happen inside the tank, as shown in Fig. 3b. To analyze the influence of the baffle's height, $h_{1} / h$ are respectively taken as $0.2,0.3,0.4,0.6,0.9$. Figs. 5 and 6 present the corresponding results. It can be clearly seen that the baffle will inhibit the rise of the disturbed wave. With an increase of the baffle height, the wave becomes lower and the period of the enveloping line shorter. When the baffle's height reaches $0.3 \mathrm{~h}$, the resonance disappears. For an increase of baffle height from 0.3 to $0.4 \mathrm{~h}$, the maximum wave elevation decreases about $10 X_{0}$. If the baffle height keeps increasing, the inhibition becomes less evident.

The case of $h_{1}=0.4 \mathrm{~h}$ is taken as an example to show the shape of the instantaneous free surface, and the period of this enveloping line is $20.992 \mathrm{~s}$ as shown in Fig. 7. In one envelope period, six time instants are selected, of which two correspond to crest (a) $t=4.677 \mathrm{~s}$, (b) $t=9.043 \mathrm{~s}$, two to trough (c) $t=9.795 \mathrm{~s}$, (d) $t=14.114 \mathrm{~s}$ and two to knot (e) $t=2.896 \mathrm{~s}$, (f) $t=9.398 \mathrm{~s}$ respectively. When the wave reaches its peak on the left wall of the tank, the free surface is just lowest on the right, and vice versa. When the free surface elevation on the two sides is very small, the value in the middle is also very small. 


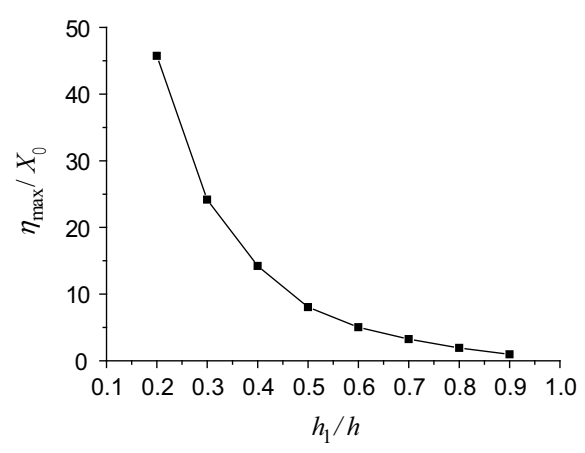

(a) Maximum wave elevation

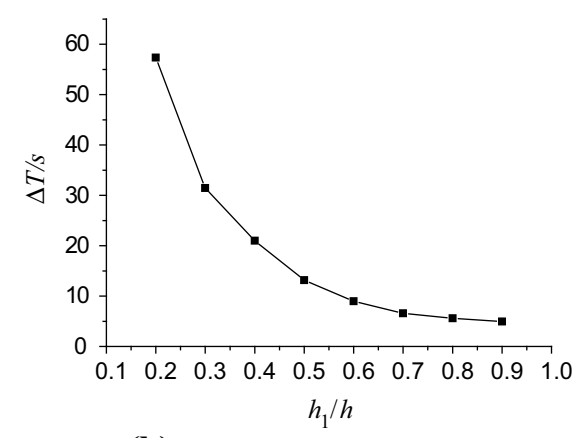

(b) Period of enveloping line

Fig. 6 The statistics of the disturbed wave with different baffle heights, $\Omega_{0}=1.002 \Omega_{1}$

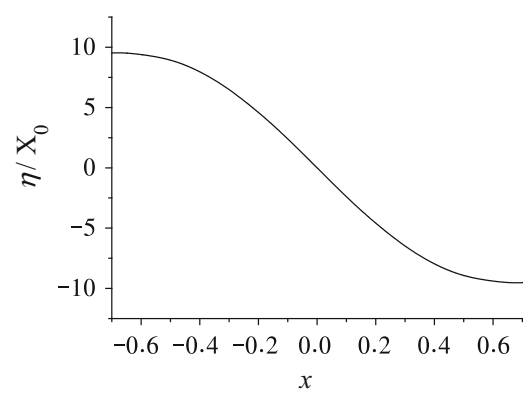

(a) $t=4.677 \mathrm{~s}$

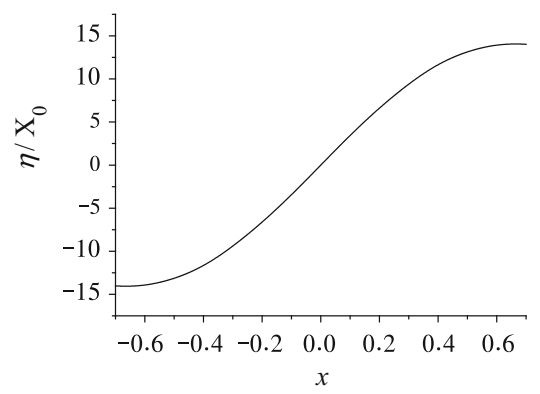

(c) $t=9.795 \mathrm{~s}$

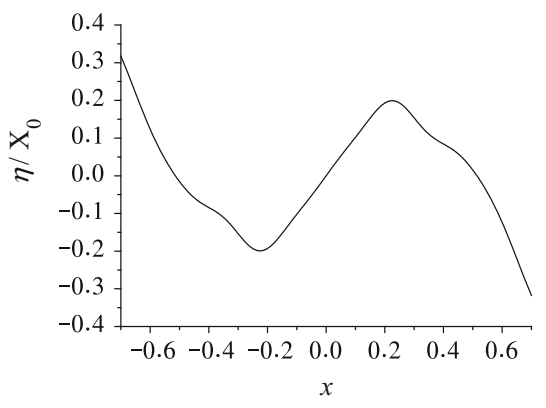

(e) $t=2.896 \mathrm{~s}$

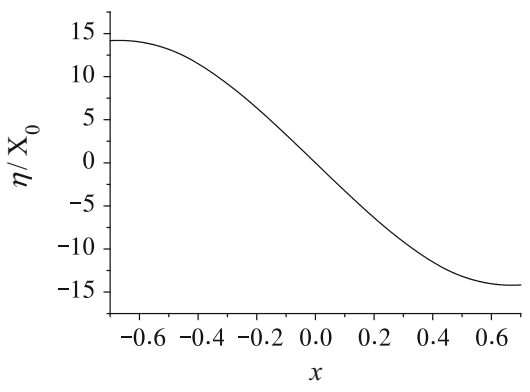

(b) $t=9.043 \mathrm{~s}$

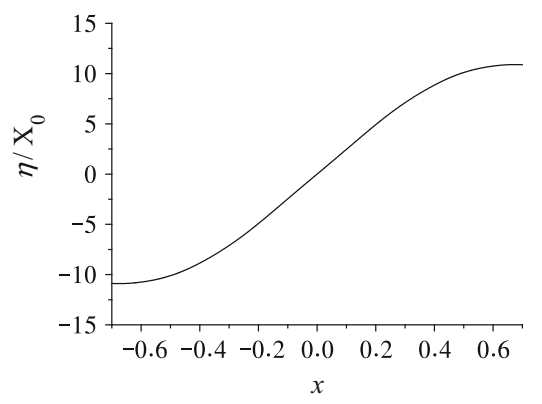

(d) $t=14.114 \mathrm{~s}$

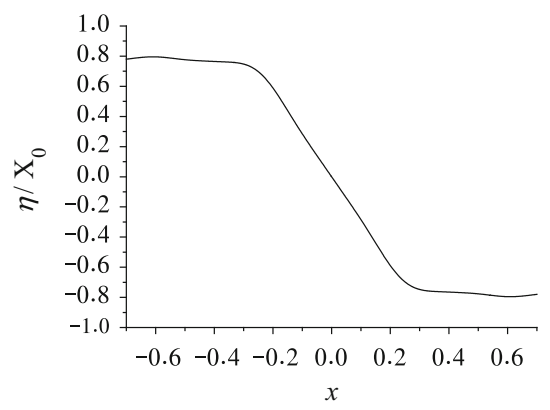

(f) $t=9.398 \mathrm{~s}$

Fig. 7 Change of free surface elevation with $x$ at different time, $\mathbf{a}, \mathbf{b}$ crest at left side, $\mathbf{c}, \mathbf{d}$ trough at left side, e, $\mathbf{f}$ knot at left side, with a fixed baffle 
5.4 Influence of baffle height on fundamental frequency

The symbol $\sigma_{n}(n=1,2,3 \ldots)$ is used to represent a series of natural frequencies of a two-dimensional rectangular tank with a baffle. $\sigma_{1}$ is the first-order natural frequency, namely the fundamental frequency. According to the formula $\Omega_{1}=\sqrt{g k_{1} \tanh k_{1} h}$, the first-order natural frequency of a rectangular liquid tank without a baffle plate can be calculated, or $\Omega_{1}=4.4910 \mathrm{rad} / \mathrm{s}$ ( $\left.a=b=h=0.7 m, k_{1}=2.244\right)$. For a liquid tank with a baffle, an initial disturbance will be exerted on it and the liquid will move at its natural frequency. Then the time history of the wave elevation is transformed by FFT to obtain the natural frequency. Fig. 8 gives the spectrum of the liquid tank with different baffle heights. Increasing the baffle's height will make the natural frequency of the system smaller. This explains why a change of the baffle can inhibit sloshing.

\subsection{Sloshing of a swaying liquid tank with a rolling baffle}

The height of the baffle cannot be easily changed after tank construction is completed. We can achieve the purpose of stabilizing the tank by adjusting the forced movement of the baffle. As linear systems satisfy the superposition principle, the movement of a tank can be decomposed into two kinds of motion. The first is the whole tank moving with velocity $U(t)=U_{0} \sin \Omega_{0} t$, which has been described in detail in Sect. 5.1 for a fixed baffle relative to the tank. Its translational equation can be set as $X(t)=-X_{0} \cos \Omega_{0} t$. The second is to point $(0,-\mathrm{h})$ as the rotating center and make a slight roll at angular velocity $\varpi(t)=\varpi_{0} \sin \left(\Omega_{\mathrm{p}} t+\varphi\right)$ under the external excitation frequency $\Omega_{\mathrm{p}}$. The symbol $\varphi$ stands for the initial phase. Suppose the equation of the roll angle with time is $\theta(\mathrm{t})=-\theta_{0} \cos \left(\Omega_{p} t+\varphi\right)$. Here, $\theta_{0}$ represents the maximum roll angle that can be reached by the baffle's movement, $\theta(t)=0$ corresponds to the vertical position and it is positive that the baffle rotates counter-clockwise.

In this paper, $\eta_{1}$ and $\eta_{2}$, respectively, represent the wave surface elevation caused by translational motion of the tank and forced oscillation of the baffle. Thus, the total free surface elevation after linear superposition can be expressed as follows:

$\eta=\eta_{1}+\eta_{2}$.

Tank parameters are still taken as $a=b=l=h=0.7 \mathrm{~m}$ and $X_{0}=2 \times 10^{-4} \mathrm{~m}$. Represented by the twodimensional rectangular liquid tank with baffle height $h_{1}=0.5 \mathrm{~h}$, it can be seen from the previous section that the natural frequency of the first-order of the liquid tank at this time is $\sigma_{1}=4.0344 \mathrm{rad} / \mathrm{s}$.

This section mainly concentrates on how to control the movement of the baffle in order to suppress the rise of the free surface in the tank and avoid the resonance that occurs the tank does translational motion and the baffle is forced to roll. We adjust the initial phase of baffle movement so that wave height $\eta_{1}$ and wave height $\eta_{2}$ are opposite, and adjust the maximum roll angle of the baffle's movement so that the amplitude of $\eta_{2}$ is almost equal to that of $\eta_{1}$. Finally, the purpose of elimination of waves can be achieved.

The tank's external excitation frequency is equal to the baffle's external excitation frequency, namely $\Omega_{0}=\Omega_{\mathrm{p}}$. Suppose $\Omega_{0}=\Omega_{\mathrm{p}}=0.997 \sigma_{1}=4.0212 \mathrm{rad} / \mathrm{s}$. The free surface elevation at $x=-b$ is plotted in Fig. 9a. The peak magnitude of the free surface elevation in each period increases with time continuously, and the liquid motion is resonant. Spectral analysis of the wave height is shown in Fig. 9b. The natural frequency $\sigma_{1}$ is equal to $4.0212 \mathrm{rad} / \mathrm{s}$.

Under the above conditions, the amplitude of cosine signal of the time history curve $\eta_{1}$ at frequency $\delta_{1}$ is calculated by Fourier series.

$a_{\omega}=\frac{2 \int_{0}^{n T} \eta(t) \cdot \cos \delta_{1} t \mathrm{~d} t}{n T}, \quad$ where $T=\frac{2 \pi}{\delta_{1}}$.

The amplitude of the sinusoidal signal can be calculated as

$b_{\omega}=\frac{2 \int_{0}^{n T} \eta(t) \cdot \sin \delta_{1} t \mathrm{~d} t}{n T}, \quad$ where $T=\frac{2 \pi}{\delta_{1}}$. 


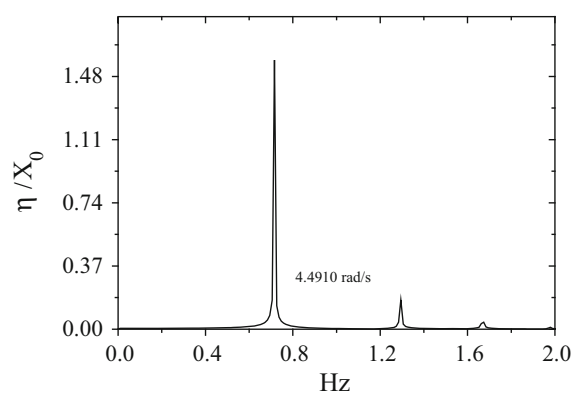

(a) $h_{1}=0, \sigma_{1}=4.491$

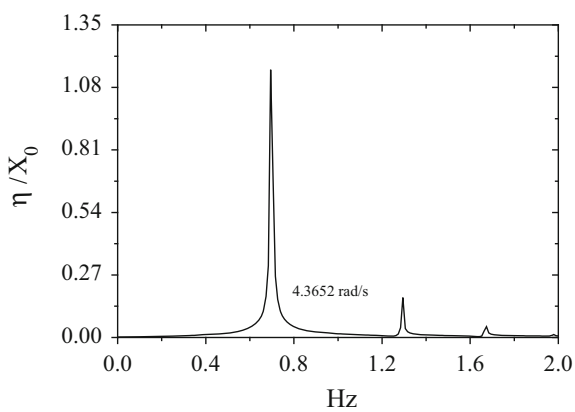

(c) $h_{1}=0.2 h, \sigma_{1}=4.3652$

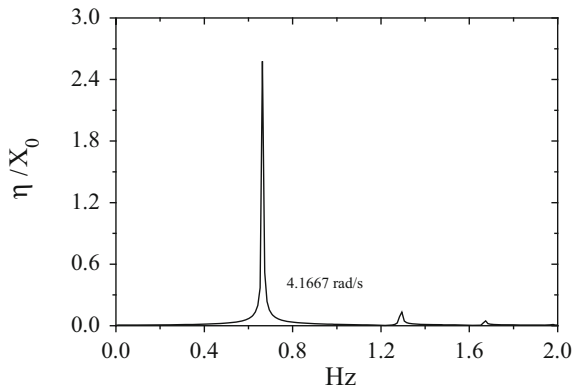

(e) $h_{1}=0.4 h, \sigma_{1}=4.1667$

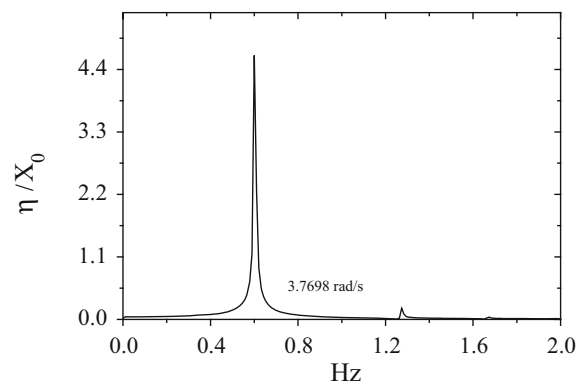

(g) $h_{1}=0.6 h, \sigma_{1}=3.7698$

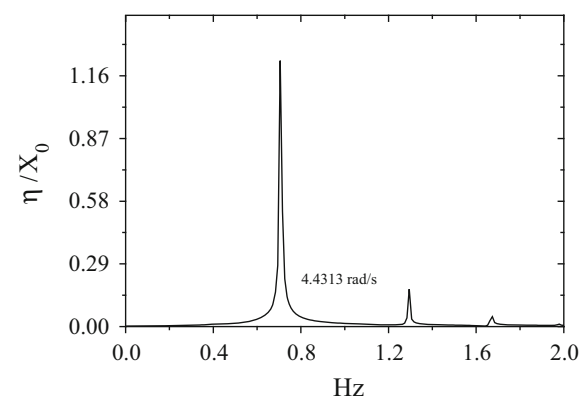

(b) $h_{1}=0.1 h, \sigma_{1}=4.4313$

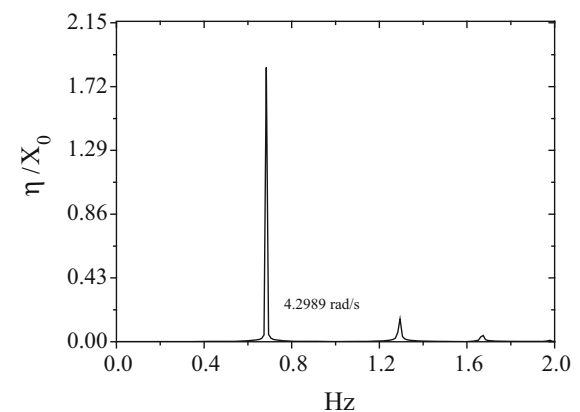

(d) $h_{1}=0.3 h, \sigma_{1}=4.2989$

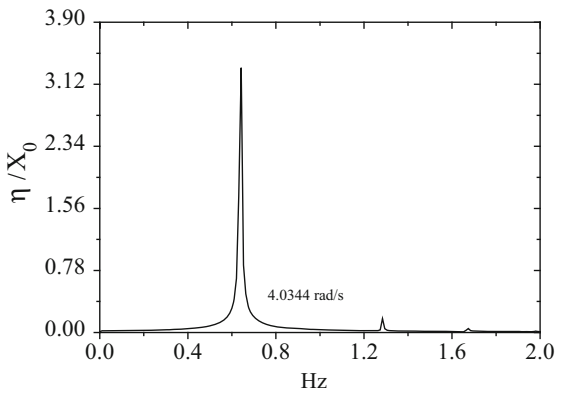

(f) $h_{1}=0.5 h, \sigma_{1}=4.0344$

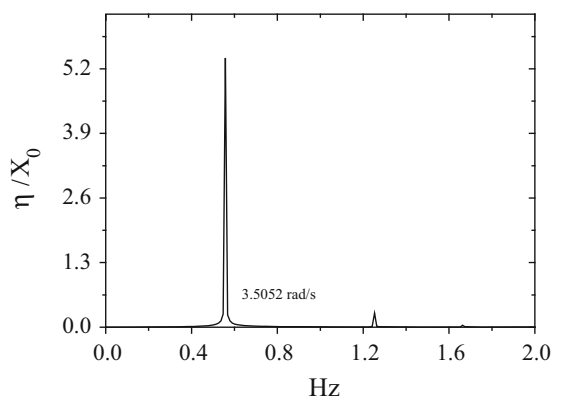

(h) $h_{1}=0.7 h, \sigma_{1}=3.5052$

Fig. 8 Spectrum of a liquid tank with a fixed baffle, baffle height $h_{1}$ changes from 0 to $0.8 \mathrm{~h}$ 


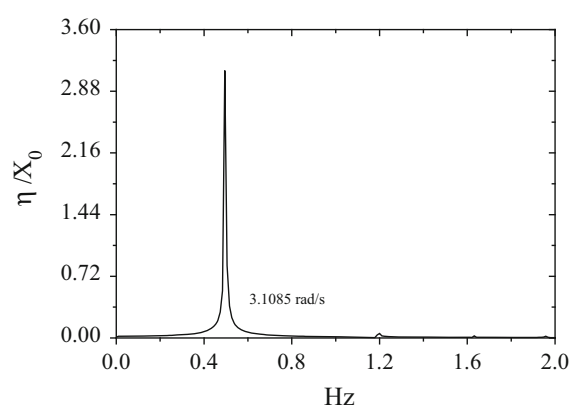

(i) $h_{1}=0.8 h, \sigma_{1}=3.1085$

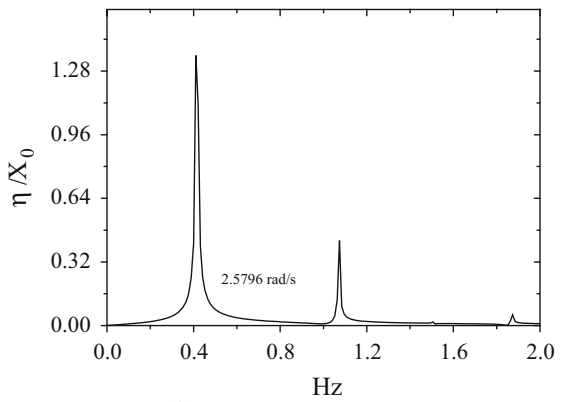

(j) $h_{1}=0.9 h, \quad \sigma_{1}=2.5796$

Fig. 8 continued
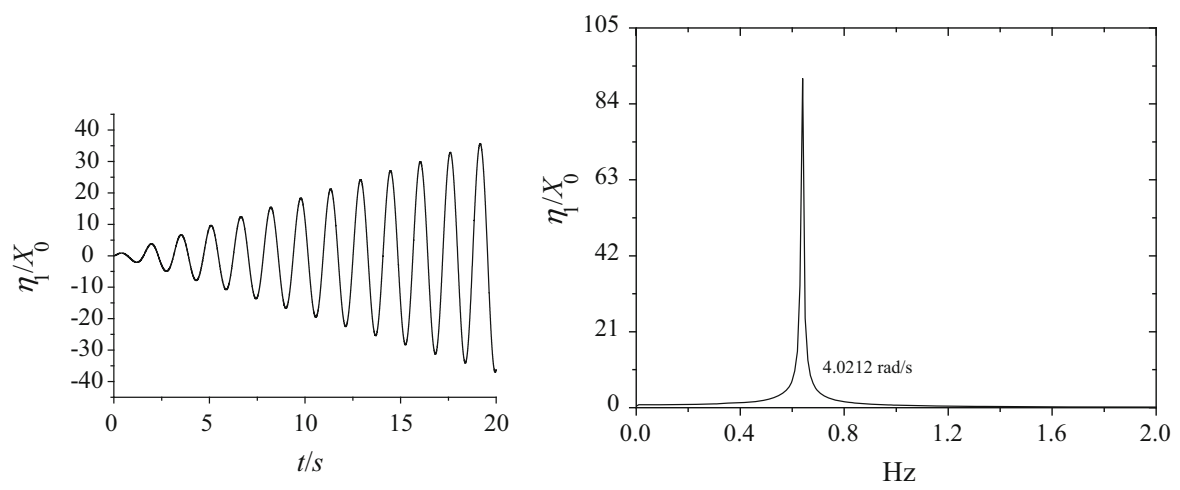

Fig. 9 Spectrum of free surface elevation $\eta_{1}, \sigma_{1}=4.0212$

The initial phase of the cosine signal whose frequency $\omega$ is:

$\varphi_{\omega}=\arctan \left(-\frac{b_{\omega}}{a_{\omega}}\right)$.

Thus, the initial phase of $\eta_{1}$ is obtained $\varphi_{\omega}=85.9023^{\circ}$. Then the baffle in the tank is forced to roll at a frequency of $4.0212 \mathrm{rad} / \mathrm{s}$. Using a similar procedure for the initial phase of $\eta_{1}$, the initial phase of $\eta_{2}$ can be calculated $\varphi_{\omega}=86.7701^{\circ}$.

After obtaining the initial phase of $\eta_{1}$ and $\eta_{2}$, the phase of $\eta_{2}$ is adjusted to be opposite to that of $\eta_{1}$. The initial phase of $\eta_{2}$ will be $\varphi=86.7701+180-85.9023=180.8678^{\circ}$. Then adjust the roll range of the baffle so that $\eta_{1} \approx-\eta_{2}$, and then $\theta_{0}=6.28 \times 10^{-3} \mathrm{rad}$. The forced motion equation of baffle plate is $\theta(t)=-6.28 \times$ $10^{-3} \cos \left(4.0212 t+180.8678^{\circ}\right)$. The total wave height $\eta=\eta_{1}+\eta_{2}$ is obtained by superimposing wave height $\eta_{1}$ and wave height $\eta_{2}$ after adjustment of initial phase and roll amplitude. As shown in Fig. 10, $\eta$ tends to be flat with extremely small amplitude, indicating that even in the case of resonance, the wave can also be eliminated by adjusting the motion of the baffle.

To see if the wave elimination is suitable for the interior part of the tank, the free surface elevation before superimposition $\eta_{1}$ and after superimposition $\eta$ is plotted. According to Fig. 10, six curves are selected which can cover the typical time instants, two of which correspond to the wave crest (a) $t=5.073 \mathrm{~s} \mathrm{(b)} t=12.894 \mathrm{~s}$, two to trough (c) $t=7.413 \mathrm{~s}$ (d) $t=13.684 \mathrm{~s}$, two to knot (e) $t=7.842 \mathrm{~s}$ (f) $t=15.631 \mathrm{~s}$. The free surface elevation $\eta_{1}$ is shown in Fig. 11, and it is almost anti-symmetric. The largest amplitude emerges at two sides, no matter whether crest, trough or knot. The eliminated wave surface is shown in Fig. 12. After superposing, the free surface shape becomes more crooked, but the elevation is extremely small. It can be concluded that the wave elevation is effective for all parts of the liquid. 
Fig. 10 Free surface elevation $\eta, \eta_{1}$ and $\eta_{2}$ by adjusting the baffle's motion

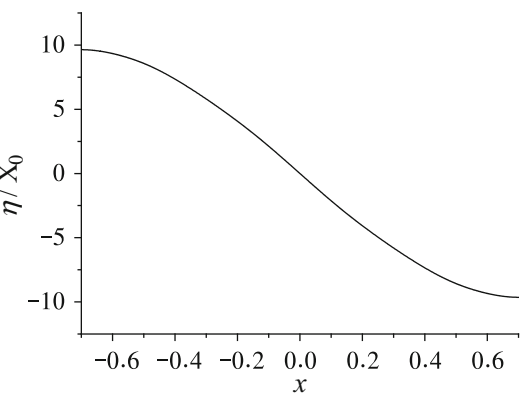

(a) $t=5.073 \mathrm{~s}$

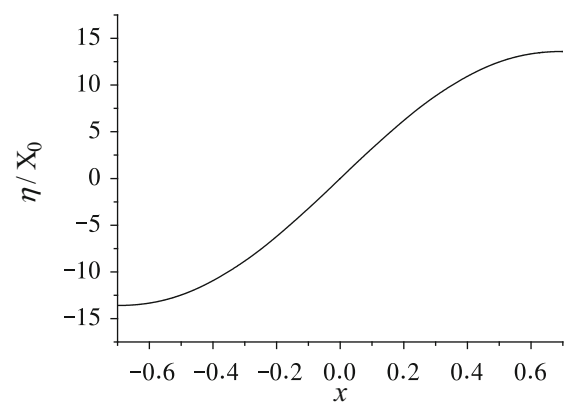

(c) $t=7.413 \mathrm{~s}$

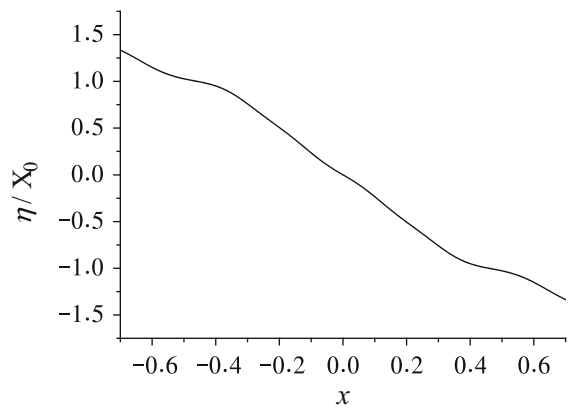

(e) $t=7.842 \mathrm{~s}$
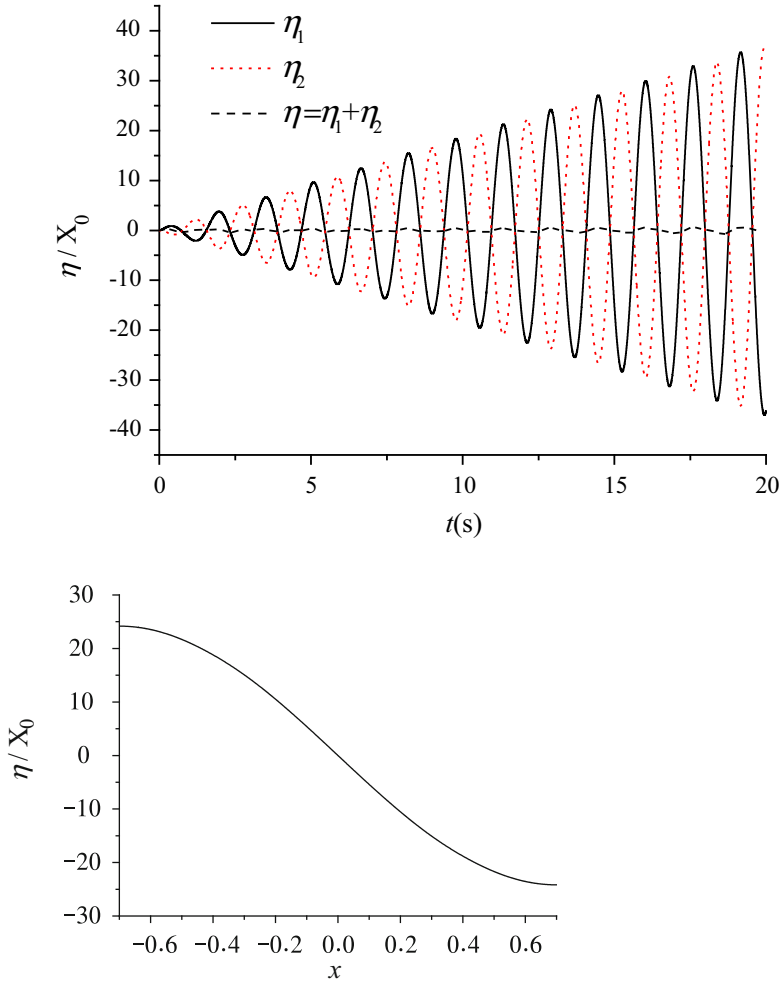

(b) $t=12.894 \mathrm{~s}$

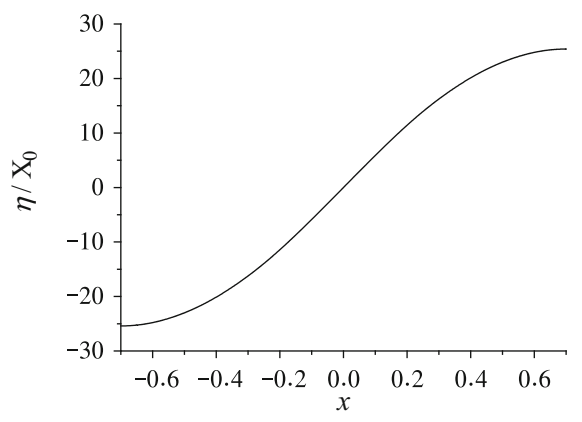

(d) $t=13.684 \mathrm{~s}$

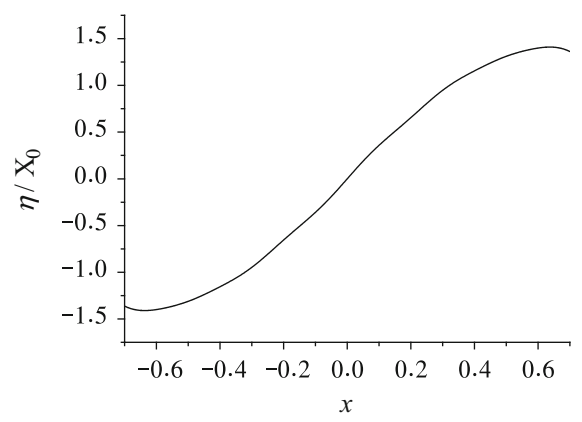

(f) $t=15.631 \mathrm{~s}$

Fig. 11 Change of free surface elevation $\eta_{1}$ with $x$ at different time, $\mathbf{a}, \mathbf{b}$ crest at left side, $\mathbf{c}, \mathbf{d}$ trough at left side, e, $\mathbf{f}$ knot at left side, with a hinged rolling baffle 


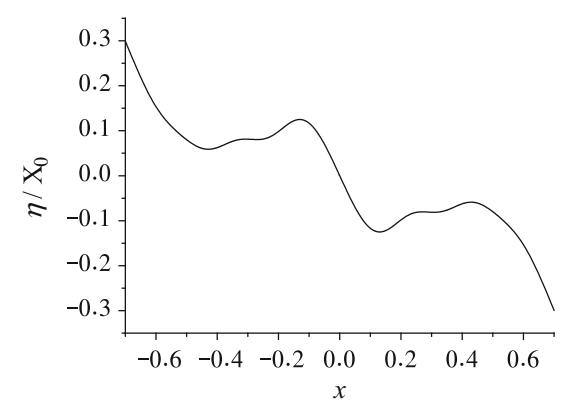

(a) $t=5.073 \mathrm{~s}$

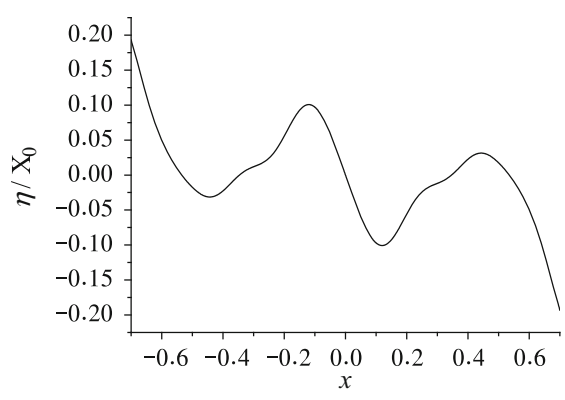

(c) $t=7.413 \mathrm{~s}$

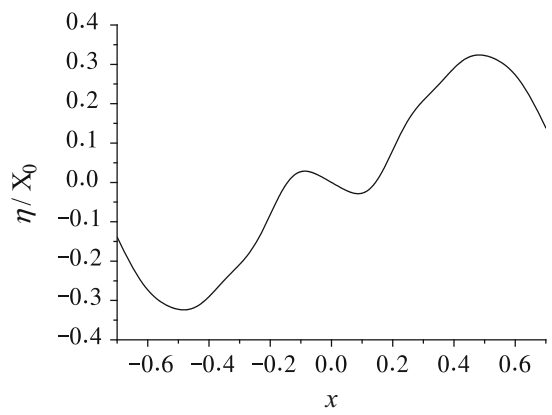

(e) $t=7.842 \mathrm{~s}$

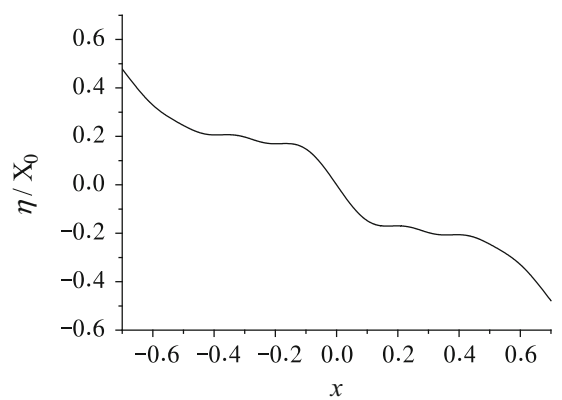

(b) $t=12.894 \mathrm{~s}$

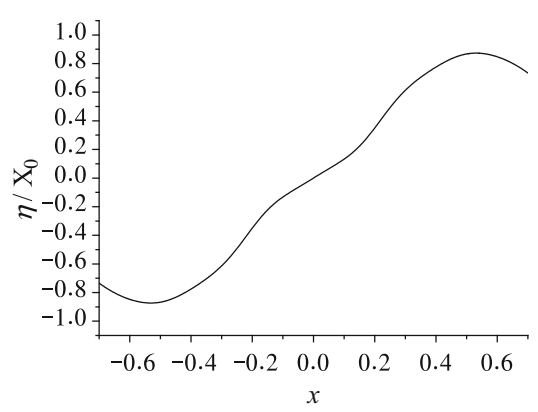

(d) $t=13.684 \mathrm{~s}$

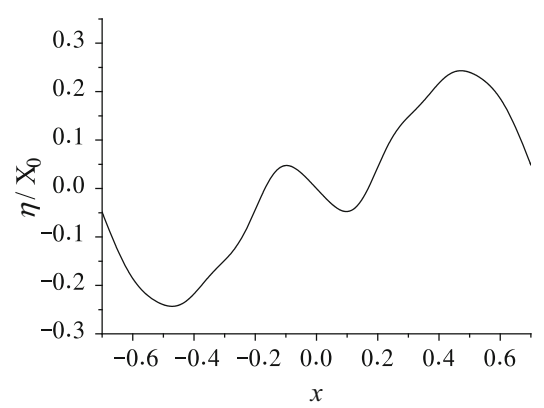

(f) $t=15.631 \mathrm{~s}$

Fig. 12 Change of free surface elevation $\eta$ with $x$ at different time, $\mathbf{a}, \mathbf{b}$ crest at left side, $\mathbf{c}, \mathbf{d}$ trough at left side, e, $\mathbf{f}$ knot at left side, with a hinged rolling baffle

The excitation frequency $\Omega_{0}=\Omega_{\mathrm{p}}=1 \mathrm{rad} / \mathrm{s}$, which is far from the natural frequency. The spectrum of $\eta_{1}(t)$ is analysed in Fig. 13a. It can be seen that the wave amplitude reaches the maximum when $\sigma_{1}=1 \mathrm{rad} / \mathrm{s}$. The corresponding phase is $\varphi_{\omega}=-0.0388^{\circ}$, while for $\eta_{2}, \varphi_{\omega}=0.0461^{\circ}$. With the above procedure, the baffle's motion is adjusted as $\theta(t)=-3.79 \times 10^{-1} \cos \left(t+180.0073^{\circ}\right)$. The free surface elevation after superimposing is given in Fig. 13b. Whether the excitation frequency is far from or adjacent to the natural frequency, the liquid motion induced by tank and baffle can be roughly balanced to inhibit the total disturbed wave.

\section{Conclusions}

A baffle was mounted at the tank bottom to inhibit sloshing; it divides the liquid tank into three regions which were solved analytically based on linear potential theory. The potential and its directional directive between different regions were continuous. Firstly, the baffle was fixed to the tank and the tank was in sway motion. The analytical 


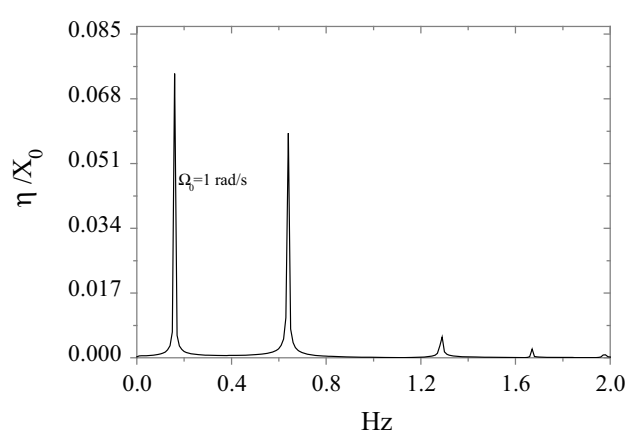

(a) Spectrum of $\eta_{1}$

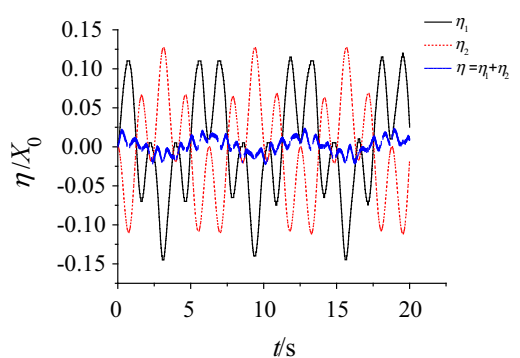

(b) Free surface elevation

Fig. 13 Free surface elevation $\eta, \eta_{1}$, and $\eta_{2}$ by adjusting baffle's motion

method was verified by comparing the present result with published data. When the excitation frequency is close to the first natural frequency of the unbaffled tank, resonance will happen. Increase of the baffle's height can inhibit the resonance, as it can make the natural frequency of the tank smaller. Then the baffle was set to roll around a hinged point. According to the superposition principle of linear systems, the movement of the liquid tank can be divided into the sway motion of the liquid tank and the roll motion of the baffle. The disturbed sloshing waves produced by two kinds of motion are summed up to obtain the total wave surface. By changing the initial phase of the forced motion of the baffle and the maximum roll angle of the baffle, the elevation of the sloshing waves generated by sway of the tank and roll of the baffle can be adjusted to be equal, and the phase to be opposite. Through this adjustment, the purpose of wave elimination can be achieved whether the frequency of sway motion is close to or far away from the resonant frequency. Under linear theory, the maximum free surface elevation is prone to emerge at two sides of the tank. Wave elimination method in the present paper is effective both for two sides as well as for the interior part of the tank.

Acknowledgements This work is supported by National Key R\&D Program of China (2018YFFC0310500) and the National Natural Science Foundation of China (Grant No. 51679045). This work is also supported by Lloyd's Register Foundation (LRF) through the joint center involving University College London, Shanghai Jiaotong University and Harbin Engineering University, to which the authors are most grateful. LRF supports the advancement of engineering-related education, and funds research and development that enhances safety of life at sea, on land and in the air.

Open Access This article is distributed under the terms of the Creative Commons Attribution 4.0 International License (http:// creativecommons.org/licenses/by/4.0/), which permits unrestricted use, distribution, and reproduction in any medium, provided you give appropriate credit to the original author(s) and the source, provide a link to the Creative Commons license, and indicate if changes were made.

\section{References}

1. Faitinsen OM (1978) A numerical nonlinear method of sloshing in tanks with two-dimensional flow. J Mar Sci Appl 22:193-202

2. Cho JR, Lee HW (2004) Numerical study on liquid sloshing in baffled tank by nonlinear finite element method. Comput Meth Appl Mech Eng 193:2581-2598

3. Cho JR, Lee HW, Ha SY (2005) Finite element analysis of resonant sloshing response in 2-D baffled tank. J Sound Vib 288:829-845

4. LA Armenio Rocca (1996) On the analysis of sloshing of water in rectangular containers: numerical study and experimental validation. Ocean Eng 23:705-739

5. Kim Y (2001) Numerical simulation of sloshing flows with impact load. Appl Ocean Res 23:53-62

6. Kim Y, Shin YS, Lee KH (2004) Numerical study on slosh-induced impact pressures on three-dimensional prismatic tanks. Appl Ocean Res 26:213-226

7. Popov G, Sankar S, Sankar TS, Vatistas GH (1992) Liquid sloshing in rectangular road containers. Comput Fluids 21:551-569

8. Wu GX, Ma QW, Eatock Taylor R (1998) Numerical simulation of sloshing waves in a 3D tank based on a finite element method. Appl Ocean Res 20:337-355 
9. Pal NC, Bhattacharyya SK, Sinha PK (1999) Coupled slosh dynamics of liquid-filled, composite cylindrical tanks. J Eng Mech 125:491-495

10. Liu DM, Lin PZ (2009) Three-dimensional liquid sloshing in a tank with baffles. Ocean Eng 36:202-212

11. Wang J, Sun SL, Hu J (2017) The coupling analysis of tank motion and sloshing by a fully nonlinear decoupling method. Nonlinear Dyn 89:971-985

12. Zhao DY, Hu ZQ, Chen G, Lim S, Wang S (2018) Nonlinear sloshing in rectangular tanks under forced excitation. Int J Nav Arch Ocean Eng 10:545-565

13. Chu CR, Wu YR, Wu TR, Wang C-Y (2018) Slosh-induced hydrodynamic force in a water tank with multiple baffles. Ocean Eng 167:282-292

14. Celebi MS, Akyildiz H (2002) Nonlinear modeling of liquid sloshing in a moving rectangular tank. Ocean Eng 29:1527-1553

15. Panigrahy PK, Saha UK, Maity D (2009) Experimental studies on sloshing behavior due to horizontal movement of liquids in baffled tanks. Ocean Eng 36:213-222

16. Abramson HN (1966) The dynamic behavior of liquids in moving containers. NASA SP-106

17. Evans DV, Mciver P (1987) Resonant frequencies in a container with a vertical baffle. J Fluid Mech 175:295-307

18. Wu GX (2007) Second-order resonance of sloshing in a tank. Ocean Eng 34:2345-2349

19. Zhou HW, Wu GX, Zhang HS (2013) Wave radiation and diffraction by a two-dimensional floating rectangular body with an opening in its bottom. J Eng Math 83:1-22

20. Zhang CW, Su P, Ning DZ (2019) Hydrodynamic study of an anti-sloshing technique using floating foams. Ocean Eng 175:62-70

21. Sygulski R (2011) Boundary element analysis of liquid sloshing in baffled tanks. Eng Anal Bound Elem 35:978-983

22. Wu CH, Faltinsen OM, Chen BF (2012) Numerical study of sloshing liquid in tanks with baffles by time-independent finite difference and fictitious cell method. Comput Fluids 63:9-26

23. Wang WY, Peng Y, Zhou Y, Zhang Q (2016) Liquid sloshing in partly-filled laterally-excited cylindrical tanks equipped with multi baffles. Appl Ocean Res 59:543-563

24. Goudarzi MA, Danesh PN (2016) Numerical investigation of a vertically baffled rectangular tank under seismic excitation. J Fluid Struct 61:450-460

25. Sanapala VS, Rajkumar M, Velusamy K, Patnaik BSV (2018) Numerical simulation of parametric liquid sloshing in a horizontally baffled rectangular container. J Fluid Struct 76:229-250

26. Sun SY, Wu GX, Xu G (2019) Free fall motion of a floating body: bubble formation and its effect. Eur J Mech B 76:178-189

27. Choun YS, Yun CB (1996) Sloshing characteristics in rectangular tanks with a submerged block. Comput Struct 61:401-413

28. Sun SY, Sun SL, Ren HL, Wu GX (2015) Splash jet and slamming generated by a rotating flap. Phys Fluids 27:1-17

29. Shi YY, Li ZF, Wu GX (2018) Motion of a floating body in a harbour by domain decomposition method. Appl Ocean Res 78:223-240

30. Zhang AM, Wang SP, Wu GX (2013) Simulation of bubble motion in a compressible liquid based on the three dimensional wave equation. Eng Anal Bound Elem 37:1179-1188

Publisher's Note Springer Nature remains neutral with regard to jurisdictional claims in published maps and institutional affiliations. 